\title{
Coriolis force in Geophysics: an elementary introduction and examples
}

\author{
F. Vandenbrouck ${ }^{\star}$, L. Berthier ${ }^{\star \star}$, and F. Gheusi ${ }^{\star \star \star}$ \\ ${ }^{\star}$ Laboratoire de Physique de la Matière Condensée, Collège de France, 11 place M. Berthelot, 75005 Paris, France \\ ${ }^{\star \star}$ Laboratoire de Physique, ENS-Lyon and CNRS, 46 allée d'Italie, 69007 Lyon, France \\ ${ }^{\star \star \star}$ Météo-France, CNRM/GMME/Relief, 42 avenue G. Coriolis, 31057 TOULOUSE Cedex, France
}

(February 2, 2008)

\begin{abstract}
We show how Geophysics may illustrate and thus improve classical Mechanics lectures concerning the study of Coriolis force effects. We are then interested in atmospheric as well as oceanic phenomena we are familiar with, and are for that reason of pedagogical and practical interest. Our aim is to model them in a very simple way to bring out the physical phenomena that are involved.

LPENSL-TH-06/2000.
\end{abstract}

\section{INTRODUCTION}

The study of mechanics in non-inertial frames, the Coriolis force effects for the rotating Earth being the paradigm, are often restricted to the standard examples of a deflected projectile and the Foucault pendulum. In these two examples, the Coriolis force is only a small perturbation. In order to make the Coriolis force effects dominant, one must consider phenomena taking place at the geophysical scale.

This is the line followed by the present paper. The first section is devoted to the presentation of the basic equations. In the second section, we discuss the Physics of anticyclones and cyclones: we describe their rotation in the geostrophic approximation, and show how cyclones (but not anticyclones) may become hurricanes. The third section presents a second example of atmospheric circulation, the Jets Streams, which are stabilized by the Coriolis force. We show also that these strong winds are associated with Rossby waves. The last section presents two examples of oceanic circulation: wind-driven circulation and Kelvin waves.

\section{BASIC EQUATIONS}

\section{A. Navier-Stokes equation in a rotating frame}

Let us introduce two frames $\mathcal{R}$ and $\mathcal{R}^{\prime}$ in relative motion. The inertial frame $\mathcal{R}$ is the geocentric one, with origin at the center $O$ of the Earth, and whose axes are along directions of fixed stars. The frame $\mathcal{R}^{\prime}$ is fastened to the Earth. It has an angular velocity $\boldsymbol{\Omega}$ with respect to $\mathcal{R}$, where $\boldsymbol{\Omega}$ is the angular velocity of rotation of the Earth $\left(\Omega \simeq 7.29 \cdot 10^{-5} \mathrm{rad} \cdot \mathrm{s}^{-1}\right)$. The following relation between the accelerations of a point $M \boldsymbol{a}_{\mathcal{R}}(M)$ in $\mathcal{R}$, and $\boldsymbol{a}_{\mathcal{R}^{\prime}}(M)$ in $\mathcal{R}^{\prime}$ may easily be obtained [1]:

$$
\boldsymbol{a}_{\mathcal{R}}(M)=\boldsymbol{a}_{\mathcal{R}^{\prime}}(M)+2 \boldsymbol{\Omega} \wedge \boldsymbol{v}_{\mathcal{R}^{\prime}}(M)+\boldsymbol{\Omega} \wedge(\boldsymbol{\Omega} \wedge \boldsymbol{O} \boldsymbol{M}) .
$$

In eq. (2.1), the term $2 \boldsymbol{\Omega} \wedge \boldsymbol{v}_{\mathcal{R}^{\prime}}(M)$ is the Coriolis acceleration, $\boldsymbol{v}_{\mathcal{R}^{\prime}}(M)$ is the velocity of $M$ in $\mathcal{R}^{\prime}$, and $\boldsymbol{\Omega}(\boldsymbol{\Omega} \wedge \boldsymbol{O} \boldsymbol{M})$ is the centrifugal acceleration. In the rotating frame $\mathcal{R}^{\prime}$, the Navier-Stokes equation takes into account the above inertial terms and reads [2]:

$$
\begin{aligned}
\frac{\partial \boldsymbol{v}_{\mathcal{R}^{\prime}}}{\partial t}+\left(\boldsymbol{v}_{\mathcal{R}^{\prime}} \cdot \nabla\right) \boldsymbol{v}_{\mathcal{R}^{\prime}}= & -\frac{1}{\rho} \boldsymbol{\nabla} p+\frac{1}{\rho} \boldsymbol{f}-2 \boldsymbol{\Omega} \wedge \boldsymbol{v}_{\mathcal{R}^{\prime}} \\
& -\boldsymbol{\Omega} \wedge(\boldsymbol{\Omega} \wedge \boldsymbol{O M})+\nu \Delta \boldsymbol{v}_{\mathcal{R}^{\prime}}
\end{aligned}
$$

The force $\boldsymbol{f}$ includes the gravitational force and other external forces if they exist, $\rho$ is the density of the fluid and $p$ the pressure field. The dependence on $M$ has been removed in all the terms for clarity. The centrifugal force is conservative. If this is also the case for $\boldsymbol{f}$, one can rewrite the terms $\boldsymbol{\nabla} p,-\boldsymbol{f}$ and $\rho \boldsymbol{\Omega} \wedge(\boldsymbol{\Omega} \wedge \boldsymbol{O} \boldsymbol{M})$ as $\boldsymbol{\nabla} p^{\prime}$, where $p^{\prime}$ is called dynamical pressure. In the rotating frame, the hydrostatic equilibrium equation is: $\nabla p^{\prime}=$ 0. The dynamical pressure $p^{\prime}$ reads, within a constant, $p^{\prime}=p+\rho g z$, where $g$ is the Earth gravity field. Recall that $g$ includes the centrifugal term, and is thus slightly different from the gravitational field, which only takes into account the Earth's attraction [1].

\section{B. Reynolds and Rossby numbers}

The nonlinearity of the Navier-Stokes equation makes it difficult to solve in general. It is hence necessary to evaluate the relative importance of the different terms in order to make further simplifications. This is done by introducing the different characteristic scales of the flow: $L$ denotes the typical spatial extension, $U$ the velocity, $\Omega$ the angular velocity and $\nu$ the kinematic viscosity. Two non-dimensional numbers may then be derived from these scales.

(i) The Reynolds number is defined as:

$$
R_{e}=\left|\frac{(\boldsymbol{v} \cdot \nabla) \boldsymbol{v}}{\nu \Delta \boldsymbol{v}}\right|=\frac{U^{2} / L}{\nu U / L^{2}}=\frac{U L}{\nu} .
$$

It characterizes the relative importance of the momentum transport in the fluid through advection and viscous diffusion. For the atmospheric flows studied here, typical values are: $U \sim 10 \mathrm{~m} \cdot \mathrm{s}^{-1}, L \sim 10 \mathrm{~km}$ and $\nu \sim 10^{-5} \mathrm{~m}^{2} \cdot \mathrm{s}^{-1}$. Thus, the Reynolds number is 
about $10^{10}$. A large value of the Reynolds number is also obtained for oceanic flows [3]. Hence, the NavierStokes equation reduces, for geophysical flows, to the Euler equation:

$$
\begin{aligned}
\frac{\partial \boldsymbol{v}_{\mathcal{R}^{\prime}}}{\partial t}+\left(\boldsymbol{v}_{\mathcal{R}^{\prime}} \cdot \nabla\right) \boldsymbol{v}_{\mathcal{R}^{\prime}}= & -\frac{1}{\rho} \nabla p+\frac{1}{\rho} \boldsymbol{f} \\
& -2 \boldsymbol{\Omega} \wedge \boldsymbol{v}_{\mathcal{R}^{\prime}}-\boldsymbol{\Omega} \wedge(\boldsymbol{\Omega} \wedge \boldsymbol{O M}) .
\end{aligned}
$$

Moreover, geophysical flows are turbulent (high Reynolds number) 泊. For the sake of simplicity, we ignore this complication in what follows. A simple way of taking into account the relevant effects of turbulence will be presented in the last section. (See section VA.)

(ii) The Rossby number is defined as:

$$
R_{o}=\left|\frac{(\boldsymbol{v} \cdot \nabla) \boldsymbol{v}}{2 \boldsymbol{\Omega} \wedge \boldsymbol{v}}\right|=\frac{U^{2} / L}{\Omega U}=\frac{U}{L \Omega} .
$$

It compares the advection and the rotation effects. The Coriolis force dominates if $R_{o} \ll 1$. A geophysical flow, characterized by a large spatial extension, may easily be influenced by the Earth's rotation, as one typically has $R_{o} \sim 10^{-2} \ll 1$. On the other hand, an emptying bathtub with $U \sim 1 \mathrm{~m} \cdot \mathrm{s}^{-1}$, and $L \sim 10^{-1} \mathrm{~m}$, has $R_{o} \sim 10^{5}$. Such a flow is more strongly influenced by the advection in the fluid, and thus by the initial conditions, than by the Earth's rotation.

\section{ATMOSPHERIC EDDIES}

\section{A. Anticyclones and cyclones}

We consider first the situation when the Rossby number is negligible. This is the case for anticyclones and cyclones since one typically has $U \sim 10 \mathrm{~m} \cdot \mathrm{s}^{-1}$, $L \sim 1000 \mathrm{~km}$, which yields $R_{o} \sim 0.1$. In the Euler equation (2.4), we only have to keep the gravity, pressure and Coriolis terms. This hypothesis constitutes the geostrophic approximation. For each point $M$ of the Earth, we define a vertical axis $(M z)$, and a cylindrical coordinate system $(r, \theta, z)$. The vertical component of the velocity field is supposed to be zero, which implies that the movements of the fluid are locally horizontal. $u$ is the radial component of the velocity field and $v$ the tangential one. The Earth's angular velocity $\boldsymbol{\Omega}$ is written as $\boldsymbol{\Omega}=\boldsymbol{\Omega}_{\|}+\boldsymbol{\Omega}_{\perp}$ where $\boldsymbol{\Omega}_{\|} \equiv \Omega \sin \lambda \boldsymbol{u}_{\boldsymbol{z}}$ and $\boldsymbol{\Omega}_{\perp}$ is $\boldsymbol{\Omega}$ 's projection on the plane $(r, \theta) ; \lambda$ is the latitude. The flow is supposed to be stationary. In this system of coordinates, the Euler equation can be rewritten, under the geostrophic approximation, as:

$$
\begin{aligned}
\frac{\partial p}{\partial r} & =\rho v f, \\
\frac{1}{r} \frac{\partial p}{\partial \theta} & =-\rho u f, \\
\frac{\partial p}{\partial z} & =-\rho g-2 \rho\left(\boldsymbol{\Omega}_{\perp} \wedge \boldsymbol{v}\right) \cdot \boldsymbol{u}_{\boldsymbol{z}} .
\end{aligned}
$$

In these equations, $f \equiv 2 \Omega \sin \lambda$ is the Coriolis parameter. In equation (3.10), the term $2 \rho\left(\boldsymbol{\Omega}_{\perp} \wedge \boldsymbol{v}\right) \cdot \boldsymbol{u}_{\boldsymbol{z}}$ is small compared to $\rho g\left(\Omega U / g \sim 10^{-5}\right)$. Equation (3.10) therefore reduces to the hydrostatic equilibrium equation $\partial p / \partial z=-\rho g$.

If we consider the case of an eddy in the Northern hemisphere and assume that the velocity field is tangential $(u=0)$, then, $v<0$ (clockwise rotation) implies $\partial p / \partial r<0$. The pressure is higher at the eddy center than outside: it is an anticyclone. A cyclone would correspond to an anticlockwise rotation. Both situations are represented in figure 1. The rotation senses are opposite in the southern hemisphere.

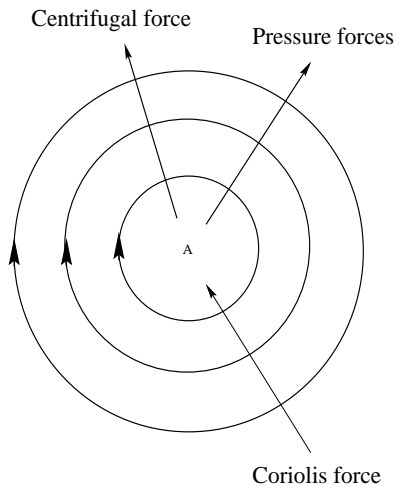

(a)

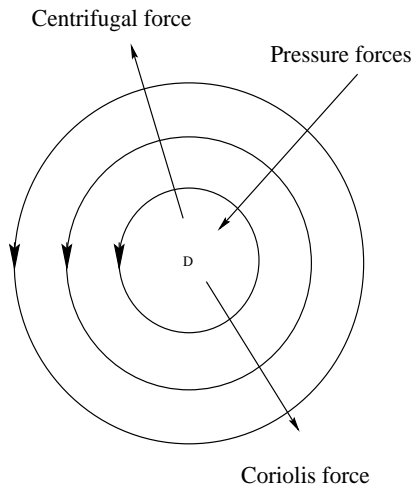

(b)
FIG. 1. Anticyclone (a) and cyclone (b) in the northern hemisphere.

We end this section with two concluding remarks about the presence of the Coriolis force:

(i) Without this force, an eddy center is always a pressure minimum. However, in the case of the anticyclone, the Coriolis force stabilizes the inverse situation, with the eddy center being a pressure maximum.

(ii) In its vectorial form, the geostrophic equilibrium equation reads: $\boldsymbol{\nabla} p^{\prime}=-2 \rho \boldsymbol{\Omega} \wedge \boldsymbol{v}$. This implies that the pressure $p^{\prime}$ is constant along a streamline. When the usual Bernoulli equation is valid, pressure variations are, on the contrary, associated with velocity variations along a streamline.

\section{B. Hurricanes}

Let us consider an eddy (anticyclone or cyclone) whose angular velocity and radius are respectively $\omega$ and $R$. The Rossby number characterizing this eddy can be written as $R_{o}=U / L \Omega=\omega / \Omega$. Therefore, the geostrophic equilibrium corresponds to a small angular velocity of the eddy, i.e. $\omega \ll \Omega$. We shall now consider the case where the eddy's angular velocity is not small compared to the Earth's rotation. This means that the centrifugal force due to the eddy rotation has to be taken into account. In this case, the Rossby number is of order unity. In the 
frame $\mathcal{R}^{\prime}$, the fluid particle has a uniform circular motion. Forces acting on it are the Coriolis force and the radial pressure gradient. The equation of motion for a fluid particle, located at the eddy's periphery reads, in $\mathcal{R}^{\prime}$ :

$$
-r_{0} \omega^{2}=-\frac{1}{\rho} \frac{\mathrm{d} p}{\mathrm{~d} r}+r_{0} f \omega,
$$

where $r_{0}$ is the eddy radius. The term $-r_{0} \omega^{2}$ corresponds to the centrifugal acceleration of the fluid particle, and $r_{0} f \omega$ is the Coriolis term.

An anticyclone in the northern hemisphere is shown in figure 1 1a. For such an equilibrium, the Coriolis force compensates both pressure and centrifugal forces. If the angular velocity of the anticyclone grows, the Coriolis force is not sufficient to counterbalance these two forces since the centrifugal force grows faster than the Coriolis force with increasing $\omega$. This is not the case for the cyclone depicted in the figure 1 $\mathrm{b}$. The pressure and centrifugal forces may counterbalance each other when the rotation of the cyclone becomes faster. This qualitative approach shows that there is no limit to the kinetic energy of rotation for a cyclone.

More quantitatively, equation (3.2) can be solved to find:

$$
\omega_{ \pm}=\frac{f}{2}\left[-1 \pm \sqrt{1+\frac{G}{G_{0}}}\right],
$$

where $G \equiv d p / d r$ and $G_{0} \equiv \rho r_{0} f^{2} / 4$. Figure 2 gives the evolution of an eddy angular velocity as a function of the radial pressure gradient. In this figure, the geostrophic situation can be found around the origin (small pressure gradient and angular velocity). In the northern hemisphere, the sign of the angular velocity is the same as that of the pressure gradient. One can even obtain the angular velocity of an eddy by developing the expression (3.3) around zero: $\omega \approx f G / 4 G_{0}$.

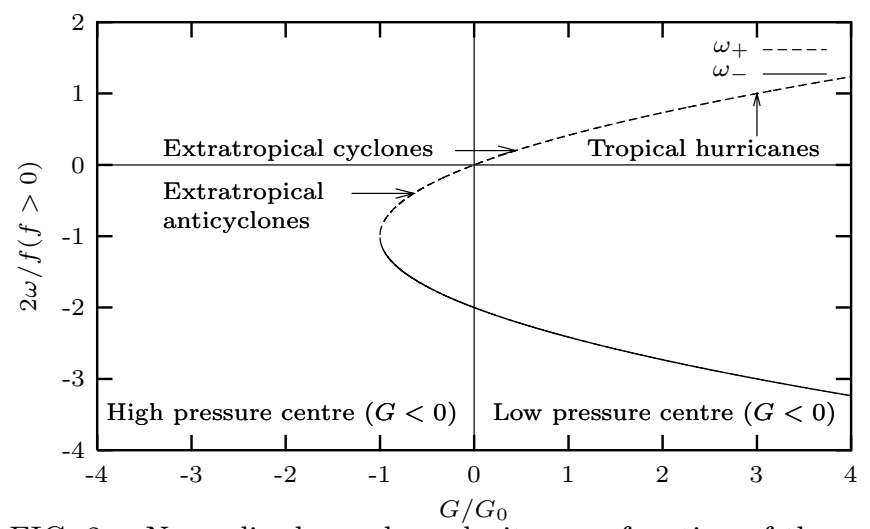

FIG. 2. Normalized angular velocity as a function of the normalized pressure gradient.

The condition $G>-G_{0}$, for the existence of the above solutions, gives a limit to the angular velocity of an anticyclone $(G<0)$. One finds $\omega_{\max }=2 \Omega \sin \lambda$. This limit does not exist for a cyclone $(G>0)$. When the angular velocity grows, the radial pressure gradient follows this evolution and becomes more and more important. This explains why hurricanes are always associated with very low pressure.

We note in conclusion that the balance between the centrifugal force and the radial pressure gradient is possible whatever the sense of rotation. Thus, the existence of clockwise hurricanes in the northern hemisphere cannot be excluded. However, most of the hurricanes observed in the northern hemisphere are anticlockwise and result from the amplification of earlier tropical cyclones, the amplification mechanism being the conversion of the latent heat of evaporating tropical warm waters into rotational kinetic energy.

\section{JET STREAMS AND ROSSBY WAVES}

The difference in solar heating between the equatorial and polar regions drives a convective cell at the planetary length scale, the Hadley cell, which extends in both hemispheres from the Equator up to the sub-tropical latitudes. The heated equatorial air rises, moves toward the poles where it cools, then sinks and comes back to the equator. When coming back, the air is deflected toward the west by the Coriolis force, generating easterlies at the tropical latitudes which are known as the Trade Winds. Conversely, the upper-troposphere trajectories toward the poles are deflected toward the east. Because of the thermal structure of the atmosphere [5], these upper-level westerlies concentrate in narrow tubes of very strong winds up to $80 \mathrm{~m} \cdot \mathrm{s}^{-1}$, the Jet Streams. The Jet Streams are typically found at altitudes of about $10 \mathrm{~km}$ and at latitudes between $30^{\circ}$ and $40^{\circ}$. However, their strength and location may depart significantly from these mean values, depending on the season, the longitude, and the day-to-day thermal structure of the atmosphere at mid latitudes. It can be noted that B. Piccard and B. Jones took great advantage of the Jet Streams for their recent travel around the world in a balloon. The Jet Streams are also useful to the planes flying from America to Europe.

In this section, we propose to show how a zonal wind (i.e. along the parallels) may be stabilized by the Coriolis force. A mass $M$ of air near the Earth's surface is reduced to a point $G$. Its coordinates are the usual spherical ones $(R, \theta, \varphi), \theta$ being the colatitude and $R$ the radius of the Earth. The velocity of $G$ can then be explicitly written: $\boldsymbol{v}_{\mathcal{R}^{\prime}}(G)=R \dot{\theta} \boldsymbol{u}_{\boldsymbol{\theta}}+R \dot{\varphi} \sin \theta \boldsymbol{u}_{\boldsymbol{\varphi}}$. The quantity $R \dot{\varphi} \sin \theta$ is the drift velocity $u_{0}$ of the point $G$ along a parallel. We deduce the following expression of the Coriolis force moment about the centre of the Earth (point $O$ ):

$$
\mathcal{M}_{O}=2 M R^{2} \Omega \dot{\theta} \boldsymbol{u}_{\boldsymbol{\theta}}+2 M R \Omega u_{0} \cos \theta \boldsymbol{u}_{\boldsymbol{\varphi}}
$$

The computation of the angular momentum of $G$ about 
$O$, in the frame $\mathcal{R}^{\prime}$, yields:

$$
\boldsymbol{L}_{\mathcal{R}^{\prime}}(O)=-\left(M R^{2}\right) \dot{\varphi} \sin \theta \boldsymbol{u}_{\boldsymbol{\theta}}+\left(M R^{2}\right) \dot{\theta} \boldsymbol{u}_{\boldsymbol{\varphi}} .
$$

The theorem of angular momentum for the point $G$, about $O$ and projected on $\boldsymbol{u}_{\boldsymbol{\varphi}}$ gives:

$$
-\ddot{\lambda}=2 \frac{\Omega u_{0}}{R} \sin \lambda
$$

where $\lambda \equiv \pi / 2-\theta$ is the latitude. This equation is linearized for small deviations around a given latitude $\lambda_{0}$, leading to

$$
\ddot{\delta}+\left[2 \frac{\Omega u_{0}}{R} \cos \lambda_{0}\right] \delta \lambda=0,
$$

where $\delta \lambda \equiv \lambda-\lambda_{0}$. The meridional motion of $G$ remains bounded, only if $u_{0}>0$, which corresponds to a drift velocity from west to east. This motion is characterized by small oscillations around the mean latitude $\lambda_{0}$ with angular frequency $\omega_{0}=\sqrt{2 \Omega u_{0} \cos \lambda_{0} / R}$. These oscillations correspond to the stationary case of a Rossby wave [6]. More generally, Rossby waves in the atmosphere are guided by strong westerlies.

\section{OCEANIC CIRCULATION}

Oceanic circulation is, of course, described by the same equations as atmospheric circulation. For large scale oceanic currents, like e.g. the Gulf stream, the geostrophic approximation (see section III A) is relevant: the Coriolis force compensates the horizontal pressure gradient, which is related to the slope of the free surface, which is not necessarily horizontal [7].

We shall be interested here in a slightly different case for which the interaction between the wind and the ocean gives rise to a current.

\section{A. Wind-driven circulation : Ekman transport}

The wind induces a friction at the ocean surface, transmitted through turbulence to the deeper layers of the sea. There is a supplementary difficulty that we cannot ignore here. The flow is not laminar, but essentially turbulent. The fluid viscosity is related to molecular agitation, dissipating the energy of a fluid particle. A diffusive momentum transport is associated with this phenomenon. In a turbulent flow, agitation dissipates the energy associated with the mean velocity of the current. This analogy allowed Prandtl to introduce the notion of an eddy viscosity 仰. In this approximation, considering $\boldsymbol{v}_{\mathcal{R}^{\prime}}$ as the mean flow velocity, the Navier-Stokes equation (2.2) remains unchanged, the eddy viscosity $\nu_{\text {turb }}$ being added to the kinematic viscosity $\nu$. It must be remarked that the former is a property of the flow while the latter is a property of the fluid. As far as geophysical flows are concerned, the kinematic viscosity is neglected, since typically $\nu_{\text {turb }} / \nu \sim 10^{5}$ for oceanic flows, and $\nu_{\text {turb }} / \nu$ is about $10^{7}$ for atmospheric flows.

Let us write the Navier-Stokes equation in projection on $(O x y z)$, where $(O x y)$ is the surface of the globe, $(O z)$ the ascendant vertical, and $(u, v, w)$ are the velocity components:

$$
\begin{aligned}
& \frac{d u}{d t}=-\frac{1}{\rho} \frac{\partial p}{\partial x}+f v+\nu_{\text {turb }}\left(\frac{\partial^{2} u}{\partial x^{2}}+\frac{\partial^{2} u}{\partial y^{2}}+\frac{\partial^{2} u}{\partial z^{2}}\right) \\
& \frac{d v}{d t}=-\frac{1}{\rho} \frac{\partial p}{\partial y}-f u+\nu_{\text {turb }}\left(\frac{\partial^{2} v}{\partial x^{2}}+\frac{\partial^{2} v}{\partial y^{2}}+\frac{\partial^{2} v}{\partial z^{2}}\right)
\end{aligned}
$$

For a stationary situation with large Rossby number, the acceleration terms are negligible: the velocity depends then only on space. The horizontal pressure gradient terms can also be neglected since the equations have been linearized and one can consider the real physical situation as the superposition of a geostrophic current (taking into account the pressure terms) and a wind-driven current, which will now be described. We consider a solution depending on space only through the coordinate $z$. The boundary conditions are the following: the velocity has to be finite both as $z \rightarrow-\infty$ and at the free surface, the stress is proportional to $\partial \boldsymbol{v} / \partial z$ and parallel to the wind flow, assumed to be in the $(O y)$ direction. One can solve eq.(5.1) and find the velocity field (the solution is straightforward defining $W(z) \equiv u(z)+i v(z))$ :

$$
\begin{aligned}
& u(z)= \pm V_{0} \cos \left(\frac{\pi}{4}+\frac{z}{\delta}\right) \exp \left(\frac{z}{\delta}\right), \\
& v(z)=V_{0} \sin \left(\frac{\pi}{4}+\frac{z}{\delta}\right) \exp \left(\frac{z}{\delta}\right),
\end{aligned}
$$

where $\delta \equiv \sqrt{2 \nu_{\text {turb }} /|f|}$ is a distance called the Ekman depth. Typical values for $\delta$ are $\delta \sim 10-100 \mathrm{~m}$. "+" stands for the northern hemisphere and "-" for the southern one.

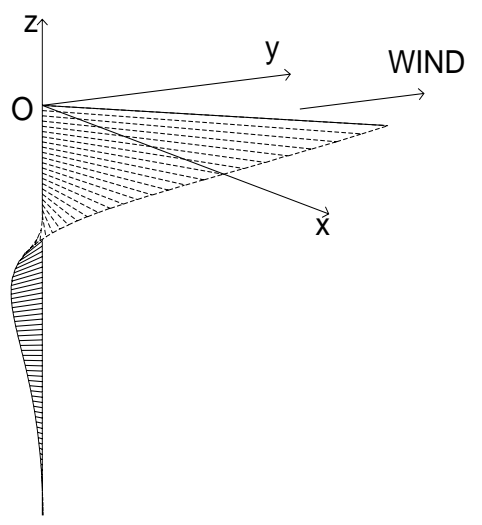

FIG. 3. Ekman spiral. The surface is generated by the velocity field $\boldsymbol{v}(0,0, z)$. 
Close to the surface $(z=0)$, the current deviates $45^{\circ}$, and the direction of the velocity rotates clockwise (anticlockwise) in the northern (southern) hemisphere. The amplitude of the velocity decreases exponentially on a length scale $\delta$, which represents the characteristic depth over which the influence of the wind is significant. This velocity field, the so-called Ekman spiral, is plotted in figure 3. The mean effect of the wind, over a depth $\delta$, is the fluid motion in a direction perpendicular to it: this effect is called the Ekman transport.

\section{B. Kelvin waves}

The main difference between atmospheric flows and oceanic flows occurs near in the coastline, limiting the waters motion. This is the origin of Kelvin waves. If one considers the deformation of the free surface of the oceans, one can see that gravity acts as a restoring force, giving rise to a "gravity wave" [8]. When influenced by the Earth's rotation, these waves are called "modified waves" [9].

Let us consider the following geometry: a south-north current, with a coast on its right (east). The coast is supposed to be a vertical wall, the water height being denoted $h_{0}+h(x, y, t)$. The Coriolis force usually deflects a south-north current toward the east, i.e. toward the coast. Hence water gathers close to the coast, and gives rise to an west-east horizontal pressure gradient counterbalancing the Coriolis force. The equations describing the gravity waves are the linearized Euler and continuity equations [8]:

$$
\begin{aligned}
& \frac{\partial u}{\partial t}=-g \frac{\partial h}{\partial x}+f v, \\
& \frac{\partial v}{\partial t}=-g \frac{\partial h}{\partial y}-f u, \\
& \frac{\partial h}{\partial t}=-h_{0}\left(\frac{\partial u}{\partial x}+\frac{\partial v}{\partial y}\right) .
\end{aligned}
$$

Taking $(O y)$ perpendicular to the coast, and considering a solution describing the above situation, i.e. $v=0$, $u=\xi(y) \exp i(\omega t-k x), h=\eta(y) \exp i(\omega t-k x)$, one obtains:

$$
\begin{aligned}
& u=u_{0} \exp \left(-\frac{f y}{\sqrt{g h_{0}}}\right) \exp i(\omega t-k x), \\
& h=u \sqrt{\frac{h_{0}}{g}}
\end{aligned}
$$

The dispersion relation is given by $\omega^{2}=g h_{0} k^{2}$, as for usual gravity waves. The characteristic length $L \equiv$ $\sqrt{g h_{0}} / f$ is the Rossby radius of deformation. At a midlatitude $\lambda \sim 45^{\circ}$, one finds $L \sim 2200 \mathrm{~km}$ for $h_{0} \sim 5 \mathrm{~km}$, while for a shallow sea, i.e. $h_{0} \sim 100 \mathrm{~m}$, one rather has $L \sim 300 \mathrm{~km}$. The surface shape generated by the Kelvin waves is plotted in figure 4 . One can notice that the surface undulation is trapped in the vicinity of the coast, and its spatial extension in the direction of the ocean is typically of order $L$.

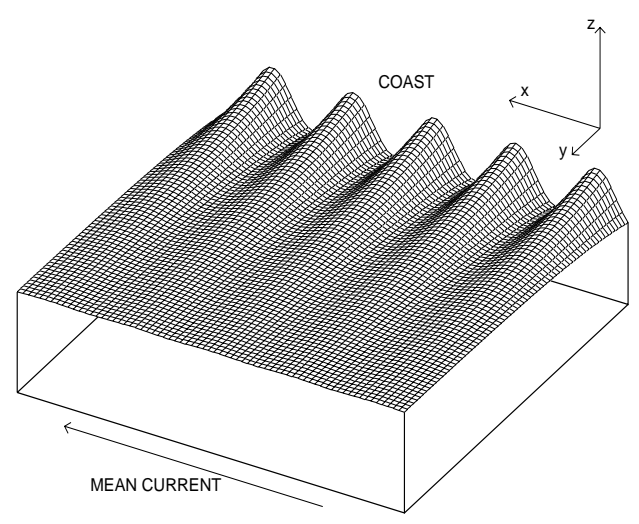

FIG. 4. Surface shape generated by a Kelvin wave.

The Kelvin waves are in fact easily observed, since the currents generated by tides are influenced by the Coriolis force and give rise to them. As a consequence, the coast is always to the right of the flow direction (in the northern hemisphere). On the oceanic basin scale, mean movements are in this case an anticlockwise rotation around a point called amphidromic point. This geometry is found in many places over the globe, the rotation being clockwise in the southern hemisphere [9].

\section{CONCLUSION}

Coriolis force effects become important as soon as the spatial extension of the flow is important $\left(R_{o} \propto 1 / L\right)$. This is the reason why the Earth's rotation considerably influences the atmosphere and oceans dynamics. We have presented in this paper several simple examples of geophysical fluid dynamics. We hope it will be helpful for Mechanics teachers to illustrate inertial effects with simple but physically relevant examples.

\section{ACKNOWLEDGMENTS}

We thank P. C. W. Holdsworth for his kind help during the preparation of the manuscript. 
[1] H. Goldstein, Classical Mechanics (Addison-Wesley, 1980), pp. 177-188.

[2] H.P. Greenspan, The theory of rotating fluids (Cambridge University Press, 1969), pp. 5-10.

[3] For oceans, $R_{e} \sim 10^{11}$, with $U \sim 1 \mathrm{~m} \cdot \mathrm{s}^{-1}, \nu \sim 10^{-6} \mathrm{~m}^{2}$. $\mathrm{s}^{-1}$ : the approximation is thus the same.

[4] M. Lesieur, Turbulence in fluids (Kluwer, 1997), pp. 315320.

[5] J.R. Holton, An introduction to dynamic meteorology (Academic Press, 1992), pp. 141-149.

[6] C.G. Rossby, Relation between variations in the intensity of the zonal circulation of the atmosphere and the displacements of the semi-permanent centers of action, J. Mar. Res., 2, (1939).

[7] Ocean circulation (The Open University, Pergamon, 1989), pp. 40-58.

[8] L.D. Landau and E.M. Lifshitz, Fluid Mechanics (Pergamon Press, 1987), pp. 31-37.

[9] S. Pond, G.L. Pickard, Introductory dynamical Oceanography (Pergamon Press, 1983), pp. 273-276. 